\title{
Editorial
}

\section{Survey of obstetric anaesthesia practice in British Columbia}

In this issue, McMorland et al. report the results of a survey of obstetric anaesthesia practices in the province of British Columbia. ${ }^{1}$ They surveyed both teaching and community hospitals and both academic and non-academic anaesthetists. Although the response rates of about 50 per cent are somewhat low, the results presented should provide food for thought for all anaesthetists who practice any amount of obstetric anaesthesia. The authors identified a number of disturbing issues, including:

- General lack of obstetrical recovery room facilities and trained recovery nurses.

- Failure to identify a designated anaesthetist to provide obstetric anaesthesia services in most of the large units.

- Universal lack of adequate pre-anaesthetic preparation in labouring patients.

- Frequent failure of physicians to remain in the hospital after establishing continuous epidural analgesia.

- Common failure to attend to technical details such as fluid preloading, lateral tilt, etc., when performing obstetric anaesthesia.

Occasional lapses in anaesthetic standards would be disconcerting enough, but the frequency of the inadequacies identified in this survey is particularly disturbing. I suspect that the problems identified by McMorland et al. and previously by Hew et al. ${ }^{2}$ are prevalent throughout Canada. At least as disturbing as the incidence of poor standards of obstetric anaesthesia practice is the fact that most of the problems identified could easily be corrected by adequately educating the physicians involved. Preanaesthetic assessment, fluid preload, oxygen sup-

From the Department of Anesthesia, University of Manitoba, Winnipeg, Manitoba, R3E 0Z3. plementation, cricoid pressure, lateral tilt and the like are all "cheap and easy" to initiate.

More difficult to deal with are the inadequacies of facilities and payment. The traditional regard for obstetric anaesthesia as being something less critical than surgical anaesthesia has given rise to the unavailability of obstetric post-anaesthesia recovery rooms (and nurses), and maternal cardiac and other monitoring equipment. Similarly, poor payment for obstetric anaesthesia services gives rise to the lack of a designated obstetric anaesthetist in larger units and failure to stay in hospital with patients receiving epidural anaesthetics in the smaller ones.

Although McMorland et al. did not include the question, I am certain they would have identified a lack of sufficient support staff for obstetric anaesthesia as well. Whereas surgical units are increasingly supplied with equipment technicians, cleanup staff and other anaesthesia support personnel, obstetric units have not followed suit. It is obvious that all anaesthetists involved in obstetric care must lobby hard to gain an equal regard for obstetric services among hospital administrators and planners as well as the paying agencies in this country.

McMorland et al. have identified two particularly disturbing practices which should be vigorously discouraged. Fully nine per cent of all the anaesthetists polled were "willing" to give a general anaesthetic by mask to an obstetric patient. Mask anaesthesia has been discouraged in this group of patients for at least 15 years, ${ }^{3}$ and is absolutely indefensible today. As well, 40 per cent of community anaesthetists were still using particulate antacids. Gibbs ${ }^{4}$ and numerous subsequent investigators have shown that the aspiration of particulate antacids is at least as dangerous as the aspiration of acid. All the correct methods of prophylaxis against acid aspiration are not yet absolute, but 0.3 molar 
sodium citrate is currently the oral antacid of choice.

Overall, the report by McMorland et al. should serve to emphasize to all obstetric anaesthetists that we still have a major task to educate anaesthetists in the community, hospital administrators, and government agencies that obstetric anaesthesia demands every bit as much attention and resources as anaesthesia for surgical procedures.

\section{Une enquête sur la pratique de l'anesthé- sie obstétricale dans Colombie Britannique}

Dans ce numéro, McMorland et al. rapportent les résultats d'une enquête sur la pratique de l'anesthésie obstétricale dans la province de la Colombie Britannique. ' Ils ont enquêté tant dans les hôpitaux communautaires que dans les hôpitaux universitaires et au niveau des anesthésistes qu'ils soient impliqués dans l'enseignement ou non. Même si le taux de réponses d'environ 50 pour cent est quelque peu bas, les résultats présentés doivent fournir matière à réflexion pour tous les anesthésistes qui pratiquent tant soit peu l'anesthésie obstétricale. Les auteurs identifient un certain nombre de points inquiétants incluant:

- L'absence généralisée de salles de réveil obstétricales et l'absence de personnel infirmier spécialement entrainé pour ces salles de réveil.

- Dans la majorité des grandes unités on ne pouvait identifier à un anesthésiste désigné spécialement afin de fournir ces services à la population obstétricale.

- L'absence universelle d'une préparation préanesthésique adéquate chez les patientes en travail.

- L'absence fréquent d'anesthésistes qui demeurent à l'hôpital après l'établissement d'une analgésie épidurale continue.

- Le manque fréquent de voir à ce que tous les détails techniques tel que précharge liquidienne, inclinaison latérale, etc ... soit accomplis lors de l'anesthésie obstétricale.

Des failles occasionnelles dans les normes anesthésiques seraient assez déconcertantes, alors que la fréquence des médiocrités identifiées dans cette enquête est particulièrement inquiétante. Je suspecte que les problèmes identifiés par McMorland et al. et au préalable par Hew et al. ${ }^{2}$ existent à travers le Canada. Tout aussi inquiétant que l'incidence des normes médiocres de la pratique de l'anesthésie obstétricale est le fait que la majorité des problèmes identifiés pourrait être facilement corrigé par une éducation adéquate des médecins impliqués. L'évaluation pré-anesthésique, la précharge liquidienne, la supplémentation d'oxygène, la pression cricoidienne, l'inclinaison latérale ainsi que d'autres sont tous faciles et peu couteux à appliquer.

La tâche sera plus difficile quant on considère le problème de locaux et de rémunération. Le concept traditionnel de considérer l'anesthésie obstétricale comme étant quelque chose de moins critique que l'anesthésie chirurgicale est responsable de la non disponibilité des salles de réveil obstétricales (ainsi que du personnel infirmier), et de l'équipement de surveillance cardiaque maternelle ou autre. De même une faible tarification des services d'anesthésie obstétricale est responsable dans les grands centres de l'absence d'anesthésiste désigné ainsi que dans les petits centres l'absence d'anesthésiste sur place après l'accomplissement de l'analgésie épidurale.

Même si McMorland et al. n'ont pas inclu la question, je suis certain qu'ils auraient identifié une absence de personnel de support suffisant pour l'anesthésie obstétricale. Alors que les unités chirurgicales sont de plus en plus assurées de techniciens de maintien et de personnel de support, les unités obstétricales n'ont pas été à la remorque. Il est évident que tous les anesthésistes impliqués dans les soins obstetricaux doivent solliciter fortement les administrateurs d'hôpitaux et les planificateurs ainsi que les responsables des tarifications de ce pays afin d'obtenir les même égards pour les services d'obstétriques.

McMorland et al. ont identifié deux pratiques particulièrement inquiétantes qui devront être fortement découragées. Neuf pour cent des anesthésistes ayant répondus à l'enquête étaient "prêts" à donner 
l'anesthésie générale au masque pour une patiente d'obstétrique. L'anesthésie au masque a été abolie pour ce groupe de patient il y a à peu près 15 ans $^{3}$ et reste indéfendable actuellement. Aussi, 40 pour cent des anesthésistes cuvrant dans les hôpitaux communautaires utilisent des antacides particulés. Le travail de Gibbs ${ }^{4}$ ainsi que d'autres investigateurs a démontré l'aspiration d'antacide particulé est pour le moins aussi dangereux que l'aspiration d'acide. Toutes les méthodes de prophylaxies contre l'aspiration d'acide ne sont pas encore absolues mais 0.3 pour cent d'une solution molaire de citrate de sodium est actuellement l'antacide oral de choix.

En résumé, ce rapport de McMorland et al. doit servir à mettre l'emphase sur tous les anesthésistes obstétricaux qu'ils ont un rôle majeur à jouer dans l'éducation des anesthésistes de la communauté, les administrateurs d'hôpitaux, des agents du gouvernements que l'anesthésie obstétricale demande autant d'attention et de ressources que l'anesthésie pourdes procédures chirurgicales.

\section{References}

1 McMorland GH, Jenkins, LC, Douglas MJ. A survey of obstetric anaesthesia practice in British Columbia. Can Anaesth Soc J 1986; 33: 185-94.

2 Hew EM, Rolbin SH, Cole AFD, Virgint S. Obstetrical anaesthesia practice in the University of Toronto affiliated hospitals and some randomly selected community hospitals. Can Anaesth Soc J 1981; 28: 158-66.

3 Smiler BG, Goldberger R, Sivak BJ, Brown EM. Routine endotracheal intubation in obstetrics. Am J Obstet Gynecol 1969; 103: 947-53.

4 Gibbs CP, Hempling RE, Wynne JW. Antacid pulmonary aspiration in the dog. Anesthesiology 1979; 51: 380-5. 